\title{
Indicadores de rendimiento para la evaluación de un servicio de bases de datos en línea
}

\author{
Luis Puente Lanzarote \\ Carlos del Campo Hilario \\ Manuel Ruiz de Luzuriaga Peña \\ Biblioteca de la Universidad Pública de Navarra
}

\subsection{Resumen}

Se presenta el modelo de evaluación del servicio de bases de datos en línea elaborado por la Oficina de Referencia de la Biblioteca de la Universidad Pública de Navarra. El modelo se inició con la definición de un conjunto de indicadores de rendimiento y se desarrolló en tres fases: recogida de datos sobre el entorno, los recursos, productos y actividades del servicio; cálculo de los valores de los indicadores - clasificados en siete categorías, a saber, economía, penetración de mercado, eficiencia, eficacia, coste-eficacia, costes y productividad —; y análisis e interpretación de los valores de estos indicadores.

Palabras clave: Evaluación. Bases de datos en línea. Indicadores de rendimiento. Control del rendimiento. Servicios bibliotecarios. Gestión de bibliotecas. Calidad. Bibliotecas universitarias. Satisfacción del usuario.

\subsection{Abstract}

An evaluation model for online database services, developed in the Universidad Pública de Navarra (Spain) library reference service, is presented. The project was initiated with the definition of a set of indicators and was developed in three stages: collection of data about the environment, resources, products and activities of the service; statistics calculation; and analysis and interpretation of the information.

Keywords: Evaluation. Online databases. Performance indicators. Performance measurement. Library services. Library management. Quality. University libraries. User satisfaction.

\section{Antecedentes y objetivo}

Este artículo presenta el resultado de un proyecto llevado a cabo en la Oficina de Referencia de la Biblioteca de la Universidad Pública de Navarra durante 
1999. Denominado ISABEL (Indicadores Sobre el Acceso a Bases En Línea), el proyecto consistió en el diseño de un sistema de evaluación para el Servicio de Acceso a Bases de Datos en Línea (SABDL) de la biblioteca universitaria.

\section{Metodología del proyecto}

El proyecto comenzó con una amplia revisión bibliográfica. En primer lugar se intentó localizar las normativas o directrices, tanto nacionales como internacionales, que hubiesen podido establecer unos criterios de evaluación precisos y comúnmente aceptados, aplicables a los SABDL. También se intentó revisar la bibliografía existente sobre evaluación, tanto sobre bibliotecas en general, como sobre servicios bibliotecarios: en especial servicios de referencia y SABDL. La revisión bibliográfica no aportó normativas, directrices ni paradigmas aceptables para la evaluación de los SABDL, por lo cual se decidió construir un modelo o marco de análisis, basándolo en el esquema input-output-outcomes, ampliamente documentado en la literatura económica y bibliotecaria. Una vez decidido el modelo de análisis se procedió a elaborar unos indicadores de rendimiento, que se integran en el mismo y lo dotan de contenido.

\section{Gestión de calidad, evaluación e indicadores de rendimiento}

En la literatura profesional se constata un interés recurrente por aplicar en las bibliotecas y servicios de información en general los principios de gestión y evaluación desarrollados a partir de modelos generados en los sectores empresariales e industriales (1). La gestión de calidad - y otros conceptos relacionados con ella, como TQM, benchmarking, etc.--, pese a un cierto carácter ambiguo y polisémico, suele ser definida en términos de planteamiento filosófico o filosofía de gestión "que impregna y reorienta todos los componentes de la organización" (García-Morales, 1995, p.10). Tiene que ver con la mejora integral de una organización, con la mejora de los procesos y los productos, con la optimación de los recursos humanos, con la eficacia interna, con la redefinición de funciones y de responsabilidades, con el establecimiento de indicadores que evalúen el nivel de satisfacción de los usuarios, con la elaboración de manuales de calidad y procedimientos, etc. Y todo ello, con el objetivo prioritario de la satisfacción del usuario, que sería la última medida de la calidad de un servicio.

El concepto de evaluación también es inconcreto y cambiante. Podemos verlo examinando algunas definiciones: "evaluación consiste en comparar lo que es con lo que debería ser" (Van House, 1990, p. 3); "estimación de la eficacia, eficiencia, utilidad y relevancia de un servicio o instalación" (UNE 50137 p. 6); "proceso que determina el mérito o valor de algo, o el resultado de ese proceso" (Scriven, 1991). Se considera, pues, evaluación el grado de cumplimiento de unos objetivos o estándares, la ponderación de algunos aspectos de un servicio, o sim- 
plemente la determinación del valor de algo. Este abanico de significados se explica por su aplicación en contextos diferentes. Así, dentro de un servicio, podemos evaluar el rendimiento, el impacto, la satisfacción del usuario, la productividad, etc. La evaluación también adquiere diferente significado según la forma o técnica que se emplee: indicadores, encuestas, cumplimiento de estándares, etc.

Del examen de la bibliografía, se pueden diferenciar varias líneas de actuación a la hora de evaluar servicios de biblioteca:

- La simple recopilación de datos estadísticos: Un ejemplo son las estadísticas de Rebiun (2), o el seguimiento de la norma UNE-EN-ISO 2789.

- Indicadores de rendimiento:Se trata de cuantificar una variable representativa de un aspecto del rendimiento del servicio. Se dispone de una serie de directrices, recomendaciones o guías generales que han impulsado el desarrollo de indicadores de rendimiento: las directrices de la IFLA (Poll, 1998), de la ALA (Van House, 1990), la norma ISO 11620 y su equivalente UNE 50137, el estudio impulsado por la DG XIII de la Comisión Europea (Ward, 1995), los indicadores de SCONUL (SCONUL, 1992), los indicadores UK OAL (King Research Ltd., 1995) para las bibliotecas públicas, la normativa ACRL (ACRL, 1994), las directrices para bibliotecas universitarias británicas (Joint Funding..., 1999) o la lista de indicadores de rendimiento de Rebiun (Grupo de Evaluación de Rebiun, 2000).

- Benchmarking: Comparación de determinados parámetros cualitativos con otros centros identificados como los que realizan las "mejores prácticas", como en Marshall (1995), Buchanan (1996), Benchmarking Team (1999) o Spiller (1998).

- Encuestas de usuarios: Se trata de conocer la opinión de los usuarios sobre determinados aspectos o la totalidad del servicio (Crawford, 2000).

- Estudios basados en las expectativas de los usuarios: Son una superación de las encuestas de usuarios, comparando la opinión de los usuarios con sus expectativas como medida objetiva de la calidad del servicio. Se está utilizando, sobre todo, la herramienta SERVQUAL (Zeithaml, 1992; Wisniewski, 1996; White, 1998; Nitecki, 1997; Coleman, 1997: Boyd, 1997; Watson, 1998; Kettinger, 1997; Pitt, 1995, 1997).

- Estudios que tratan de evaluar el impacto de las bibliotecas, universitarias generalmente, en la consecución de los fines de la institución que las sustenta, como, por ejemplo, el de Lindauer (2000).

Junto a esta variedad de propuestas, también se detecta una serie de tendencias que, sea cual sea el modelo que se aplique, se van imponiendo en la evaluación de los servicios bibliotecarios: 
- Abandono del modelo clásico de evaluación basado en análisis de los productos (outputs), y su sustitución por un análisis de los resultados (outcomes) o grado de cumplimiento de los objetivos.

- Se tiende a valorar aspectos cualitativos, frente al anterior predominio de los aspectos cuantitativos.

- Se tiene más en cuenta al usuario y al entorno informativo.

- Apoyos y colaboraciones con otras disciplinas como la estadística, la economía, la gestión empresarial, etc.

No obstante, a pesar de las indefiniciones terminológicas, apenas enunciadas, y de la diversidad de propuestas metodológicas que pueden documentarse, como ha señalado, por ejemplo, R. Cullen (1999, p.12-13), las cuestiones metodológicas son menos significativas que el hecho de que las organizaciones se están esforzando en su propia evaluación, vinculándola a la mejora de la calidad de sus servicios, a la toma de decisiones y a la asignación de recursos, retroalimentándose de sus esfuerzos evaluadores. En este contexto, la evaluación del rendimiento de un servicio puede considerarse como un componente de la implantación de un sistema de gestión de calidad, que permitiría conocer de forma cuantitativa el grado de cumplimiento de unos objetivos marcados (Poll, 1998). Otras propuestas metodológicas consideran la evaluación del rendimiento en un sentido más genérico, equiparándola a lo que se entiende por gestión de calidad, e incardinándola en un enfoque de toma de decisiones. Esta es, por ejemplo, la perspectiva utilizada en el proyecto CAMILE (Blagden, 1997) (3).

Nuestra propuesta sigue el primer enfoque: Consideramos la evaluación como una parte del proceso de mejora y gestión de la calidad en los servicios. A su vez, nos hemos decidido por un tipo concreto de evaluación: la evaluación del rendimiento a través de indicadores de rendimiento. Esta elección ha venido determinada por una serie de factores:

- La evaluación a través de indicadores de rendimiento es la opción mejor documentada, tanto en el ámbito teórico como normativo, así como a través de estudios llevados a la práctica.

- Esta opción permite cumplir una serie de condicionantes que nos planteamos a priori: que la recogida de datos y el mantenimiento y análisis de los mismos se puedan realizar dentro del propio servicio; que el proyecto se pueda aplicar de forma inmediata; que el modelo de evaluación pueda repetirse de forma casi rutinaria en años posteriores; que no suponga una carga excesiva para el servicio; y que no sea demasiado exigente para los usuarios.

A pesar de esta elección, hemos seguido atentamente la literatura sobre otros modelos de evaluación, en especial la evaluación del servicio basada en las 
expectativas del usuario (SERVQUAL) y los incipientes estudios sobre la medición del impacto de los servicios. La decisión de no seguir estas vías ha estado motivada por su escasa implantación y documentación, y las excesivas exigencias para el servicio y los usuarios. No obstante, recomendamos un atento seguimiento del desarrollo de estos modelos en el futuro.

Por último, señalar que los indicadores de rendimiento que se han desarrollado hay que encuadrarlos en un contexto, fuera del cual algunos pueden perder sentido y significado; este contexto es un SABDL (teledocumentación, según otras denominaciones), que presenta las siguientes características:

- Está insertado en el servicio de referencia de una biblioteca universitaria.

- El personal que atiende el SABDL no se dedica en exclusiva a él.

- Tiene carácter complementario respecto de los otros servicios prestados en el servicio de referencia.

- Está dirigido sólo a una parte de la comunidad universitaria.

- Las búsquedas las realizan los bibliotecarios.

\section{Modelo de Evaluación}

El propósito inicial del proyecto que estamos presentando era el de proporcionar unos instrumentos que sirviesen para evaluar el rendimiento de un SABDL y para la toma de decisiones referidas al mismo. El modelo de evaluación por el que optamos, como ya se anunció antes, se basa en la definición de unos indicadores de rendimiento, que nos ofrecerán información sobre el funcionamiento del servicio y sobre su eficacia.

Un indicador de rendimiento puede definirse, siguiendo las directrices de la IFLA, como una "proposición cuantificada utilizada para evaluar y comparar el rendimiento de una biblioteca en el cumplimiento de sus objetivos", especialmente definidos "en términos de necesidades de los usuarios" (Poll, 1998, p. 20).

Como ya se comentó anteriormente, nuestra comunicación no presenta y no es equiparable a un sistema de gestión de calidad. Este es un concepto que implicaría más aspectos que los que aquí se tratan, y que debiera superar el ámbito de un servicio tan circunscrito como el nuestro y enmarcarse, al menos, en un estilo de gestión de toda la Biblioteca.

Partimos también del criterio de que no existe una correspondencia unívoca entre indicadores de rendimiento y evaluación. En la literatura, a veces, se usan como equivalentes los términos medida del rendimiento y evaluación, pero pensamos que se debe distinguir entre la descripción, de la que formaría parte la medida del rendimiento, y los juicios de valor, que son los que conforman la evaluación (Cotta-Schønberg, 1995). En la misma línea que lo indicado por C. Abbott (1994, 
p. 9), consideramos que los indicadores de rendimiento contribuirán al proceso de evaluación del servicio, pero "evaluació"n es un término más amplio que designa la valoración del rendimiento. Las directrices de la IFLA también apuntan en este sentido cuando, al tratar sobre la interpretación de las medidas de rendimiento, junto con otras consideraciones, señalan que los indicadores únicamente pueden mostrar un rendimiento alto o bajo, pueden quizá ayudar a identificar las causas de un rendimiento bajo, pero "a menudo, será necesario una investigación adicional para encontrar las causas" (Poll, 1998, p. 26).

Nuestro modelo de indicadores de rendimiento no es tampoco equiparable al cumplimiento de unas normas preestablecidas. En parte de la literatura consultada, cuando se desarrollan indicadores de rendimiento, se señala la necesidad de establecer una serie de objetivos para, posteriormente, evaluar los indicadores obtenidos de acuerdo con la consecución o no de estos objetivos prefijados. Estos objetivos se han denominado "estándares de ejecución satisfactoria" (Salgueiro, 1998). No obstante, éste no es el único planteamiento documentado, y no siempre se establece una equiparación a priori de los indicadores de rendimiento con normas $\mathrm{u}$ objetivos preestablecidos. Ésta es, por ejemplo, la tesis que sustenta C. Abbott, para quien, aunque hay una conexión entre indicadores de rendimiento y normas, la relación debe ser creada en la etapa de interpretación, no al comenzar la tarea; y añade que: "embarcarse en el desarrollo de indicadores de rendimiento con unas ideas preconcebidas de normas u objetivos preestablecidos, puede ser desorientador y contraproducente" (Abbott, 1994, p. 9). Utilizando una línea de argumentación similar, Cotta-Schønberg (1995), en el sistema de evaluación diseñado para la Copenhagen Business School Library, relega a una tercera fase la cuestión de si los resultados obtenidos corresponden a unos objetivos prefijados. Tras una primera fase dedicada a la medida del rendimiento (“¿qué estamos haciendo?"), una segunda de evaluación (“¿lo estamos haciendo bien?”), le sigue una tercera de diseño estratégico (“¿estamos haciendo lo que queremos hacer?”), en la que se formularían claramente los criterios de valor objetivos a utilizar, ya sean normas o estándares, niveles de "mejores prácticas" o metas por alcanzar. Su modelo acaba con una última fase de acción correctora (“¿qué queremos cambiar?”). Si los resultados son negativos, hay tres vías para la corrección: revisar las propias medidas de rendimiento, replantearse los objetivos y reforzar o cambiar los procedimientos.

Incluso en aquellos casos en los que se señala la necesidad de fijar objetivos preestablecidos a los indicadores, no se proporcionan ni ejemplos ni pautas para determinarlos. De los diferentes modelos analizados el que mejor integra, en un marco conceptual homogéneo, los diversos elementos que entran en juego (estadísticas, relaciones entre ellas, indicadores), es el propuesto por C. Abbott (1994) (4).

Partimos de considerar una serie de estadísticas referidas a varios aspectos: el entorno o contexto en que se desarrolla el servicio; los recursos de que dispo-

Scire. $7: 1$ (en.-jun. 2001) 89-114. 
ne (inputs); los productos de las operaciones y actividades bibliotecarias (outputs); el uso que los usuarios dan a estos productos y el grado de satisfacción con los mismos (resultados, outcomes); y el impacto del servicio. Estos elementos interactúan entre sí con distintos tipos de relaciones: económicas, de penetración de mercado, de eficiencia, de eficacia, de coste-eficacia, de costes, de productividad y de impacto. Estas relaciones se cuantifican por medio de unos indicadores de rendimiento. El esquema que puede ilustrar el marco general (estadísticas y relaciones entre ellas) en que se basa esta propuesta de indicadores de rendimiento es el que aparece en la figura 1.

El modelo de evaluación que proponemos consta de tres fases. Se inicia con la recogida de datos del funcionamiento del servicio, lo que permitirá medir la actividad del mismo, no así todavía su rendimiento. Estaríamos en una fase meramente descriptiva, destinada a obtener información sobre las distintas variables que conforman el servicio. En una segunda etapa, se procederá al procesamiento de estos datos para obtener los valores o resultados de los indicadores previamente definidos, que nos ofrecerán información sobre el rendimiento y eficacia del servicio. Finalmente se trataría de analizar e interpretar estos valores; en definitiva, de proceder a la evaluación del servicio.

Sin un umbral que nos marque el cumplimiento, o no, de unos objetivos para cada indicador, éstos nos proporcionarán evidencias sobre las que basar los jui-

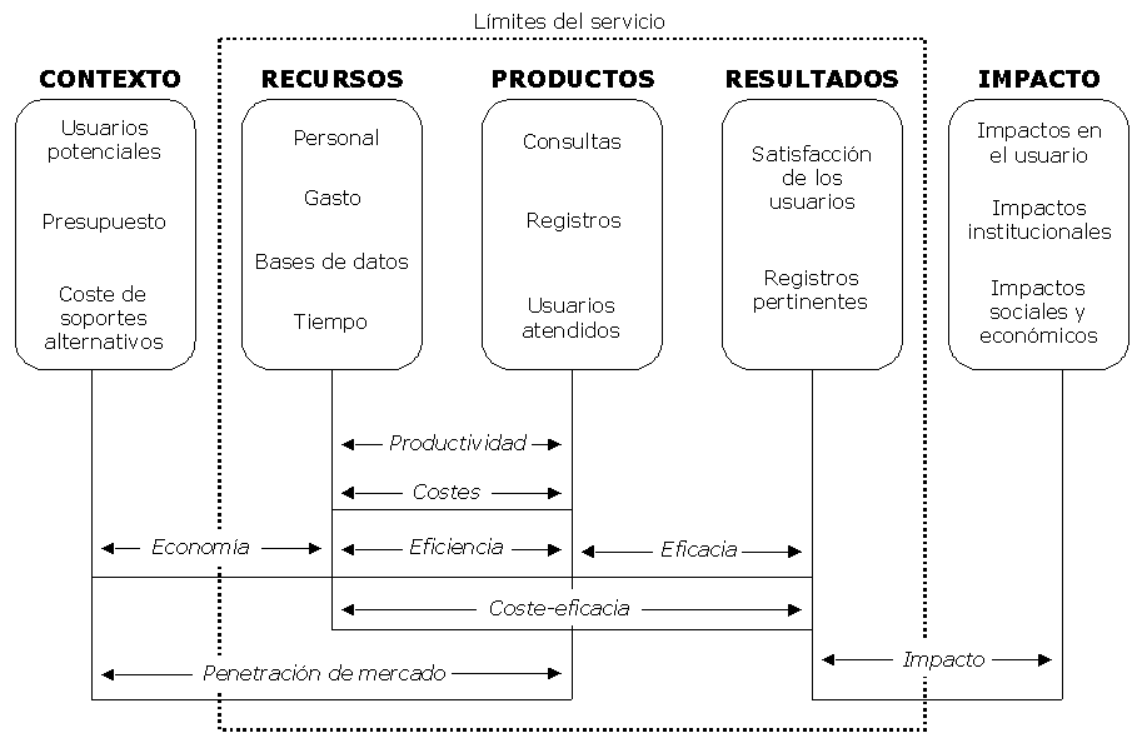

Figura 1: Esquema adaptado del modelo propuesto por C. Abbott. (1994, p. 19)

Scire. $7: 1$ (en.-jun. 2001) 89-114. 
cios sobre el rendimiento del servicio, pero no serán sustitutos de esos juicios. A partir de los indicadores se necesitará interpretarlos antes de que pueda sustantivarse la evaluación.

\subsection{Estadísticas que es necesario recopilar}

Se han definido cuatro grupos de estadísticas de interés, a saber:

- Estadísticas referidas al contexto en el que se desarrolla el servicio: Son medidas externas al ámbito del servicio que se pretende evaluar, pero que son necesarias para contextualizar los datos del mismo. Medidas de este tipo son los usuarios potenciales, el presupuesto de la unidad en la que está integrado el servicio o el coste de suministrar en otros soportes alternativos las bases de datos que se utilizan.

- Estadísticas referidas a los recursos que gestiona el servicio: Son los recursos materiales y humanos que utiliza el servicio. Las medidas que se tendrán en cuenta son el personal que atiende el servicio, las bases de datos utilizadas, el gasto del servicio, el intervalo de tiempo transcurrido desde la formulación de las consultas hasta su resolución, y el tiempo efectivo que ocupa la realización de las consultas

- Estadísticas referidas a los servicios, productos o actividades: Son el producto generado por la actividad llevada a cabo en el servicio. No tienen en cuenta su calidad ni la adecuación a unos objetivos determinados. Podríamos denominarlos como la producción bruta del servicio, e incluyen aspectos como el número de consultas, de registros obtenidos en las mismas o de usuarios atendidos.

- Estadísticas referidas a los resultados del servicio: Representan la medida en la que el servicio cumple con sus objetivos; en este caso, la satisfacción del usuario. Incluyen medidas de la satisfacción del usuario sobre la consulta en general, del número de referencias pertinentes de entre las recuperadas y de la satisfacción del usuario con el tiempo de entrega de los resultados.

\subsection{Método de recopilación de los datos}

La recopilación de las estadísticas que hemos denominado como "referidas al entorno en que se desarrolla el servicio" se obtienen del propio contexto institucional: otros servicios de la biblioteca, la dirección de la misma, u otras unidades de la Universidad.

Las estadísticas ligadas directamente al servicio, a las búsquedas que gestiona, se recopilan básicamente en tres momentos diferentes a lo largo de cada una de las transacciones de búsqueda efectuadas: la entrevista entre el usuario y el bibliotecario encargado de realizar la búsqueda, la propia realización de la bús- 
queda por parte del bibliotecario y una encuesta de satisfacción que cumplimentarán los usuarios con posterioridad a la entrega de los resultados de la búsqueda.

En un servicio que no atienda un número excesivo de consultas, se pueden recoger los datos de todas las consultas. Sin embargo, en aquellos SABDL con gran volumen de transacciones, se pueden estudiar métodos de muestreo, para que la recopilación de datos no suponga una carga excesiva para el servicio ni para los usuarios (5).

\subsection{Indicadores de rendimiento}

Las medidas, o datos estadísticos, por sí solos, no son especialmente relevantes, pero relacionadas entre sí generan los indicadores de rendimiento. Los indicadores que proponemos (que se explican con detalle en el siguiente apartado) se agrupan, según el tipo de relación que se establece entre los distintos elementos del servicio, de la siguiente forma:

1) Indicadores de macronivel: Son indicadores que consideran el servicio como un todo y lo comparan con su entorno.

a) Indicadores de economía: Reflejan la relación entre los recursos y el contexto, e intentan medir los recursos asignados al servicio, teniendo en cuenta el entorno en que se desarrolla el mismo.

Indicador 1. Gasto relativo

Indicador 2. Gasto relativo por base de datos

b) Indicadores de penetración de mercado: Reflejan la relación entre los productos y el contexto. Intentan cuantificar en que medida la comunidad a la que se pretende servir "consume" los productos ofrecidos. Indicador 3. Penetración de mercado

4) Indicadores del nivel del servicio: Son los que, dentro del esquema que utilizamos, mejor reflejan el rendimiento del servicio.

a) Indicadores de eficiencia: Relacionan recursos y productos, señalando la correcta asignación de recursos a una operación. El ejemplo paradigmático es la rapidez en suministrar el servicio, en nuestro caso, en presentar el resultado final de una consulta. Estos indicadores no se deben confundir con los de costes y productividad, que son indicadores de rendimiento operacional y, aunque también relacionan productos y recursos, intentan medir otras cuestiones distintas.

Indicador 4. Tiempo por consulta

Indicador 5. Intervalo de transacción

Indicador 6. Ocupación temporal del servicio

Scire. $7: 1$ (en.-jun. 2001) 89-114. 
b) Indicadores de eficacia: Relacionan resultados y productos. Tratan de medir la manera en que los productos ofrecidos cumplen los objetivos propuestos, en nuestro caso la satisfacción del usuario.

Indicador 7. Satisfacción del usuario

Indicador 8. Registros pertinentes

Indicador 9. Satisfacción con el intervalo de transacción

c) Indicadores de coste-eficacia: Relacionan resultados y recursos. Su objetivo es cuantificar el coste que supone alcanzar un determinado nivel de eficacia.

Indicador 10. Gasto por consulta satisfactoria

Indicador 11. Gasto por registro pertinente

3) Indicadores de rendimiento operacional: Son los indicadores que reflejan los aspectos "empresariales" del servicio, relacionando los productos obtenidos con los recursos invertidos. Expresan la relación entre lo producido por el servicio y los recursos que se necesitan para llegar a dichos niveles de producción.

a) Indicadores de costes: Relacionan recursos y productos, e intentan medir el coste económico de los productos.

Indicador 12. Gasto por consulta

Indicador 13. Gasto por registro

b) Indicadores de productividad: Relacionan recursos y productos, e intentan medir la producción generada según los recursos disponibles. Indicador 14. Consultas por bibliotecario

\subsection{Impacto}

La evaluación del impacto del servicio, o lo que es lo mismo, el medir las consecuencias beneficiosas de su uso, debiera ser el punto final de todo modelo de evaluación. El impacto de un servicio puede considerarse en tres niveles de trascendencia progresiva:

- Lo primero que sería necesario encontrar son aquellas medidas significativas que permitiesen conocer el verdadero valor del servicio para el usuario, es decir, el beneficio que éste obtiene como consecuencia del uso del servicio.

- En segunda instancia habría que demostrar el valor añadido que se aporta en el contexto institucional, en la consecución de sus propósitos y objetivos. En nuestro caso, como servicio de apoyo a la docencia e investigación, y, por tanto, el impacto que tiene en los resultados docentes y de investigación. 
- Por último, sería interesante analizar el propio impacto de la investigación, a través de sus expresiones (publicaciones, patentes, etc.) yde las repercusiones, económicas, sociales, etc., que aquella produce.

En el nivel individual, ciertos indicadores ya apuntados, y que definiremos en el siguiente apartado, pueden identificarse con atributos determinantes y generadores de valor añadido o beneficio para el usuario (tiempo de respuesta, coste, relevancia). Pero en general, la evaluación de cualquier servicio bibliotecario desde la perspectiva de los resultados obtenidos o del impacto producido en la comunidad universitaria y en la sociedad en su conjunto presenta múltiples dificultades: resultados difusos, intangibles, difíciles de identificar y de cuantificar. Dificultades e indefiniciones que exceden los propósitos de este proyecto, por lo que en esta propuesta se obvia la evaluación del impacto del servicio.

\subsection{Interpretación de los indicadores}

La tercera fase de la evaluación, consiste en el análisis e interpretación de los indicadores o "proposiciones cuantificadas" del rendimiento, para así determinar el nivel de calidad del servicio. Los indicadores, incluso cuando no se hayan definido con unos objetivos previos que permitan compararlos con los resultados, proporcionan información útil para mostrar la calidad del servicio. En primer lugar, nos muestran la situación del rendimiento del servicio, y son de utilidad tanto en el ámbito interno - de la Oficina de Referencia o de la Bibliotecacomo externo — referido al marco universitario-, al poder informar a la comunidad universitaria sobre un servicio de la biblioteca y mostrar unos resultados del mismo. En segundo lugar, permitirán un análisis longitudinal: comparar las variaciones del rendimiento del servicio, y de los diferentes factores que lo componen, a lo largo de los años. Pueden utilizarse también para análisis transversales, comparándolos con indicadores de servicios similares de otras bibliotecas. Para que estas comparaciones sean posibles, es necesario que, además de los propios indicadores, los servicios que se quieran comparar y su entorno, tanto la biblioteca en su conjunto como la universidad, sean de características similares.

En el supuesto de obtener unos rendimientos deficientes o disonantes, se necesitaría un análisis adicional para determinar sus causas y proponer, en su caso, las medidas de actuación pertinentes. En estos supuestos, habrá que tener en cuenta tanto los datos que sugiera el propio indicador como su análisis en relación con otros indicadores. Incluso en algunos casos será necesario algún tipo de investigación posterior.

En la definición pormenorizada de cada uno de los indicadores que proponemos, abordada en el siguiente apartado, se apuntarán las vías de interpretación particularizadas de los mismos. 


\section{Indicadores de rendimiento}

Para la exposición de los indicadores se ha utilizado el marco descriptivo propuesto en la norma UNE 50137:2000, asumiendo además como propias las definiciones propuestas en ella. Únicamente se ha prescindido del apartado "indicadores relacionados", opcional en la propia norma, pues las relaciones entre éstos ya se han indicado en la figura 1, donde se exponen más claramente dentro del esquema general que explica el modelo propuesto.

\subsection{Gasto relativo}

- Objetivo: Medir qué parte de los recursos del servicio de referencia acapara el SABDL.

- Campo de aplicación: El indicador es válido para bibliotecas cuyo SABDL forme parte del servicio de referencia. Cuando el SABDL sea un servicio independiente, se podría considerar el gasto del servicio respecto al gasto total de la biblioteca, considerando en ambos casos gastos de personal, mantenimiento e infraestructuras, puesto que serían independientes, y no compartidos con otros servicios. La comparación transversal, lógicamente, deberá hacerse entre bibliotecas que usen el mismo tipo de datos.

- Definición: Porcentaje del gasto directo del servicio de referencia que supone el gasto directo del SABDL, siendo el gasto directo del servicio de referenci el coste de las adquisiciones y las suscripciones, y el gasto directo del SABDL el coste de las suscripciones, conexiones y documentos obtenidos.

- Método: Se obtiene el gasto directo del SABDL en un año natural, agregando los costes de las diversas consultas y suscripciones. Se obtiene el gasto directo del servicio de referencia en un año natural, agregando el coste directo de las búsquedas en línea, el coste de las suscripciones (digitales e impresas) y el coste de las adquisiciones (digitales e impresas). El valor del indicador es (A/B) x 100\%, siendo A el gasto directo del SABDL y B el gasto directo del servicio de referencia.

- Interpretación: La interpretación estará supeditada a los objetivos y filosofía de la biblioteca: si es un servicio complementario, que se utiliza para satisfacer las consultas residuales que no estén cubiertas por el resto de recursos del servicio de referencia, habría que estudiar los valores altos, e incluso tomar medidas, como, por ejemplo, la suscripción de nuevas bases de datos en CD-ROM. Unos valores muy bajos, por contra, pudieran indicar desconocimiento o poca aceptación del servicio. De todas formas, para hacer un diagnóstico acertado, hay que contrastarlo con otros indicadores, como la penetración de mercado (indicador 3), la satisfacción del usuario (indicador 7) o los registros pertinentes (indicador 8). 
- Fuentes (6): Abbot 1994 * (Proportion of budget committed).

\subsection{Gasto relativo por base de datos}

- Objetivo: Controlar que en ninguna base de datos se realicen unos gastos que superen el coste de una forma de acceso alternativa.

- Campo de aplicación: En principio, todos los SABDL.

- Definición: Número de bases de datos cuyo gasto supere el precio de una forma de acceso alternativa.

- Método: Se controla el gasto realizado para cada base de datos (se excluyen los gastos de proveedor, como cuota de alta o suscripciones). Seguidamente, se investigan los costes que supondría una forma de acceso alternativa (CD-ROM, un acceso en línea diferente...) a las bases de datos que se han utilizado, y que no estén ya suscritas en otro formato. Del mismo modo, se investigan aquellas bases de datos ya suscritas en otro formato, sobre las que se hayan realizado consultas en línea para períodos o partes no cubiertas con la suscripción.

El valor del indicador sería Sxi, siendo $\mathrm{x}=1$ si $\mathrm{A}>\mathrm{B}, \mathrm{y} \mathrm{x}=0$ si $\mathrm{A}<\mathrm{B}$, entonces $\mathrm{x}=0$, donde $\mathrm{A}$ es el gasto de una base de datos y $\mathrm{B}$ es el gasto que supondría una forma de acceso alternativa a la misma base de datos.

- Interpretación: El indicador serviría para detectar un uso antieconómico del servicio: si el valor del indicador es superior a cero, esto supondría que hay alguna base de datos para la que habría que estudiar una forma de acceso alternativa.

\subsection{Penetración de mercado}

- Objetivo: Medir qué parte de la población-objetivo del servicio lo usa realmente.

- Campo de aplicación: El indicador es válido para bibliotecas cuyo SABDL esté dirigido a una población limitada y mensurable, como pueda ser el caso de la población investigadora de una comunidad universitaria.

- Definición: Porcentaje de usuarios reales respecto a los usuarios potenciales, siendo los usuarios reales los que han usado efectivamente el servicioy los usuarios potenciales la población a la que está dirigido el servicio.

- Método: Se obtiene el número de usuarios diferentes que han usado el servicio en un año, así como el número de usuarios potenciales del servicio al principio del año de referencia. El valor del indicador es (A/B) x 100\%, siendo $\mathrm{A}$ el número de usuarios reales y $\mathrm{B}$ el número de usuarios potenciales. 
- Interpretación: Unos valores bajos pueden indicar desconocimiento del servicio o poca aceptación. Para determinarlo será necesario un estudio y una comparación con otros indicadores. Puede ser interesante una desagregación por unidades universitarias para obtener una visión más real de la situación. Para la comparación entre diferentes instituciones, más que la semejanza de tamaño, será necesaria la homogeneidad de las definiciones de usuario potencial. De todas formas, para afinar en la interpretación del indicador, es necesario conocer los hábitos investigadores y las necesidades de información de los supuestos usuarios potenciales. Esto se puede llevar a cabo mediante encuestas que determinen esos hábitos y necesidades o contrastando el uso del SABDL y el de otros servicios de información que pueda ofrecer la biblioteca (bases de datos en CD-ROM, DSI, bases de datos de sumarios, servicios de acceso al documento, etc.).

- Fuentes (6): Abbott $1994 *$ (Actual users as proportion of potential users), De la Mano 1998* (Índice de uso del servicio), Poll 1998 ** (Penetración de mercado), UNE 50137 ** (B.2.1.1 Porcentaje alcanzado de la población destinataria), Ward 1995 ** (D.11 Active library users per capita), King Research Ltd. $1995 * *$ (Usuarios como proporción de la población).

\subsection{Tiempo por consulta}

- Objetivo: Determinar el tiempo efectivo que se emplea en la resolución de las consultas.

- Campo de aplicación: Todos los SABDL en los cuales la búsqueda se realice por intermediación de un bibliotecario.

- Definición: Mediana de los tiempos efectivos empleados en la resolución de las consultas, siendo el tiempo efectivo el sumatorio de los tiempos reales empleados en la consulta.

- Método: En cada consulta distinguimos cinco fases: a) información previa y entrevista; b) preparación de la búsqueda; c) consulta en línea; d) preparación del documento con los resultados; e) entrevista posterior con el usuario. Se anotan los tiempos efectivos empleados en cada fase para todas las consultas efectuadas en un año, expresados en horas y minutos. La suma de los tiempos efectivos de cada fase es el tiempo efectivo total de una consulta. Se ordenan los tiempos efectivos de las consultas y se calcula la mediana, que es el valor del indicador.

- Interpretación: Los valores del indicador anormalmente altos deberían corregirse. Para ello se desagregará el indicador en las diferentes fases de consulta, para restringir más la ubicación del problema. Unos valores bajos deberían confrontarse con los indicadores de eficacia. Si la eficacia es bue- 
na y los tiempos bajos, tendríamos un servicio eficiente. Si los tiempos son bajos y la eficacia también, se podría pensar que lo uno es consecuencia de lo otro. Un análisis longitudinal nos permitiría apreciar mejor la interrelación de estas dos variables.

- Fuentes (6): Abbott 1994 * (Time taken per search).

\subsection{Intervalo de transacción}

- Objetivo: Medir la rapidez con que se satisfacen las consultas.

- Campo de aplicación: En principio, todos los SABDL.

- Definición: Mediana de los intervalos de transacción, siendo éstos los tiempos totales transcurridos desde que se solicita la consulta hasta que están dispuestos los resultados.

- Método: Se anota el día, la hora y el minuto en que se solicita la consulta. Se anota el día, la hora y el minuto en que se avisa al usuario de que los resultados están disponibles. Se restan ambas medidas, expresando el resultado en horas y minutos. Seguidamente, se ordenan los intervalos de transacción y se calcula la mediana, que es el valor del indicador.

- Interpretación: Para interpretar los resultados de este indicador deberíamos considerar si los valores suponen un tiempo de espera razonable. Se debería confrontar este indicador con los valores del indicador 9 (satisfacción con el intervalo de transacción) para intentar determinar cuál puede ser ese tiempo razonable. Para corregir los valores del indicador, deberíamos fijarnos en factores como tiempos de tramitación, disponibilidad del personal, tiempos muertos entres fases de la consulta, etc. De todas formas habría que controlar que unos tiempos por consulta (indicador 4) extraordinariamente altos, no tengan un peso tal que distorsione la interpretación de este indicador.

- Fuentes (6): Abbott 1994 * (Time taken per search), Ward $1995 * * *$ (Overall speed in satisfying request).

\subsection{Ocupación temporal del servicio}

- Objetivo: Medir qué parte del tiempo total de trabajo ocupa la prestación del SABDL.

- Campo de aplicación: Este indicador tiene sentido en bibliotecas en las cuales el SABDL no suponga una dedicación exclusiva del personal que lo atiende.

- Definición: Porcentaje del tiempo efectivo empleado en atender las consultas del SABDL respecto al tiempo total de trabajo del personal que atiende el servicio, definiéndose el tiempo efectivocomo en el indicador 4 ,.y sien- 
do el tiempo total de trabajo aquél que permanece en el trabajo el personal que atiende el SABDL, atendiendo diversas labores.

- Método: Se obtiene la suma de los tiempos efectivos empleados en resolver las consultas de un año (indicador 4), y se obtiene el tiempo total de trabajo del personal que atiende el SABDL. El valor del indicador es (A/B) x 100\%, siendo A el tiempo efectivo de las consultas y B el tiempo total de trabajo.

- Interpretación: El valor de este indicador tiene que marcar el volumen de trabajo que genera el SABDL, y estar en relación con los indicadores 3 y 14.

\subsection{Satisfacción del usuario}

- Objetivo: Medir el grado de satisfacción del usuario con el servicio en general.

- Campo de aplicación: En principio, todos los SABDL.

- Definición: Nivel medio de satisfacción del usuario con el servicio, expresado en una escala de 1 a 5.

- Método: Al finalizar cada consulta, se entregará al usuario un cuestionario, en el que se le pide que califique la transacción en general en una escala de 1 a 5 , siendo 5 muy satisfactoria y 1 completamente insatisfactoria. Se suman todas las calificaciones recibidas. El valor del indicador es A/B, siendo A la suma total de las calificaciones y B es el número de consultas calificadas por los usuarios.

- Interpretación: Como todos los indicadores subjetivos, debe manejarse con mucho cuidado. En primer lugar, se debería determinar si las contestaciones al cuestionario son suficientes para obtener conclusiones. Si el grado de satisfacción no se considera adecuado, deberán investigarse las causas, incluso analizando individualmente las transacciones que han recibido calificaciones muy bajas; en primer lugar confrontándolo con los indicadores 8 y 9, para determinar si alguno de ellos nos revela cual es el factor determinante de la insatisfacción (las referencias o el intervalo de transacción). También habrá que fijarse en las observaciones formuladas por los usuarios para aislar otros factores de insatisfacción, e incluso se pueden desarrollar encuestas especiales: pudiera ser que la insatisfacción viniera determinada por otros factores, como el entorno de la entrevista, poca amabilidad de los bibliotecarios, que no guste el formato en que se entregan las referencias, etc. En principio, el indicador sólo servirá para dar la voz de alarma ante una situación no deseada; su solución vendrá determinada por posteriores investigaciones.

- Fuentes (6): Abbott 1994 * (Surveys of needs fill rate), Van House 1990 * (15 Online search evaluation: c Overall satisfaction with the online search), King Research Ltd. 1995 * (Satisfacción del usuario con...), Poll 
$1998 * *$ (16 Satisfacción del usuario), UNE 50137** (B.1.1.1 Satisfacción del usuario).

\subsection{Registros pertinentes}

- Objetivo: Medir la satisfacción del usuario con los documentos ofrecidos.

- Campo de aplicación: En principio, todos los SABDL.

- Definición: Porcentaje de registros pertinentes sobre el total de registros suministrados, siendo los registros pertinentes aquéllos considerados como tales por el usuario, y definiendo registro como el documento en formato completo. No se onsideran los formatos abreviados ni los listados.

- Método: Al finalizar cada consulta, se entregará al usuario un cuestionario (7), en el que se le pedirá que califique cada registro suministrado como: a) registros muy importantes, el valor de la búsqueda sería menor sin ellos; b) registros pertinentes pero de menor importancia, no obstante, está bien disponer de ellos; c) registros muy importantes, o simplemente pertinentes, pero que ya conocía; d) registros pertinentes, pero de valor marginal, la búsqueda hubiese sido igual de valiosa sin ellos; e) registros nada pertinentes. Los registros calificados como a) y b) se consideran pertinentes, y los calificados como c), d) y e) como no pertinentes. Finalmente, se tabulan todas las calificaciones recibidas por los registros suministrados en un año. El valor del indicador es (A/B) x 100\%, siendo A el número de registros considerados pertinentes y $\mathrm{B}$ el número de registros calificados por los usuarios.

- Interpretación: El conocimiento de este indicador nos permitirá conocer la precisión de las búsquedas. La precisión es una medida indirecta del tiempo y del esfuerzo que le cuesta al usuario manejar la documentación que le suministra el servicio (Baker, 1991, p. 287), por lo tanto, también es una medida indirecta de la satisfacción: a menor tiempo y esfuerzo del usuario, mayor satisfacción. Este indicador no pretende medir la habilidad del personal que realiza la búsqueda, pues la precisión, considerada en este sentido, no tiene ningún valor por sí misma sin conocer el grado de exhaustividad, cuyo cálculo es inviable en el contexto en el cual nos movemos. Es difícil establecer cuál sería un mínimo aceptable (8). La misma presencia de respuestas que califiquen los resultados como no pertinentes, debería llevar aparejada una investigación de las causas. Todo ello sin perder de vista la relación con el indicador 7, que señala la satisfacción general respecto a las consultas.

- Fuentes (6): Van House 1990 * (15 Online search evaluation by Satisfaction with the search product), Massísimo 1994 **** (Satisfacción del usuario con...), King Research Ltd. 1995 *** (Satisfacción del usuario con...). 


\subsection{Satisfacción con el intervalo de transacción}

- Objetivo: Medir la eficacia del servicio desde el punto de vista de su prontitud.

- Campo de aplicación: En principio, todos los SABDL.

- Definición: Nivel de satisfacción del usuario con el intervalo de transacción, expresado en una escala de 1 a 5 y definido como en el indicador 5.

- Método: Al finalizar cada consulta, se le entregará al usuario un cuestionario, en el que se le pedirá que califique el intervalo de la transacción en una escala de 1 a 5 , siendo 5 muy satisfactorio y 1 completamente insatisfactorio. Se suman todas las calificaciones recibidas. El valor del indicador es $\mathrm{A} / \mathrm{B}$, siendo A la suma total de las calificaciones y $\mathrm{B}$ el número de consultas calificadas por los usuarios.

- Interpretación: El valor del indicador hay que confrontarlo con el de satisfacción general (indicador 7). Se podría derivar un estudio sobre el nivel de satisfacción según el plazo en que se han entregado los resultados.

- Fuentes (6): King Research Ltd. 1995 ** (Satisfacción del usuario con los niveles de características), Cram $1993 * *$ (User satisfaction by attribute levels: ... such as timeliness...), Massísimo 1994 *** (Satisfacción con el tiempo de respuesta).

\subsection{Gasto por consulta satisfactoria}

- Objetivo: Medir el coste medio que supone un servicio eficaz.

- Campo de aplicación: En principio, todos los SABDL.

- Definición: Gasto directo del SABDL —definido como en el indicador 1dividido entre el número de consultas satisfactorias ponderadas, considerándose así las que, en el cuestionario que se entrega al usuario (ver indicador 7) son calificadas con 4 ó 5.

- Método: Se obtienen el gasto directo del SABDL en un año natural (véase indicador 1), el número de consultas satisfactorias en un año natural, el número total de consultas realizadas en el mismo períodoy el número de consultas calificadas por los usuarios (véase indicador 7). El valor del indicador es $(\mathrm{AxD}) /(\mathrm{BxC})$, siendo A el gasto directo del SABDL, B el número de consultas satisfactorias, $\mathrm{C}$ el número total de consultas y $\mathrm{D}$ es el número de consultas calificadas por los usuarios.

- Interpretación: Como indicador de coste-eficacia, nos marca el nivel de gasto que supone un servicio satisfactorio. El valor del indicador puede mejorarse, o reduciendo costes o aumentando la eficacia; sin embargo, nosotros consideramos, siguiendo a Gorman (Gorman 1999), que "en un 
servicio de información, la eficacia es un bien mayor que la eficiencia". Por lo tanto, una mejora en este indicador, sería conveniente realizarla por la vía de aumentar la eficacia, o, en el caso de producirse por la vía de reducir costes, si esto no supusiera un descenso sustantivo de la eficacia. Una posibilidad de este indicador sería la de calcular una "frontera de posibilidades" de la eficacia: el momento en que el aumento de costes no suponga ya un aumento de la eficacia. Esa "frontera" nos indicaría que no podemos aumentar la eficacia aumentando el gasto, y tendremos que recurrir a mejorar las otras variables que conforman la eficacia.

- Fuentes (6): King Research Ltd. 1995 ** (Coste medio/nivel de satisfacción del usuario), Massísimo $1994 * * *$ (Coste por nivel de satisfacción del usuario), Lancaster $1996 * * * *$

\subsection{Gasto por registro pertinente}

- Objetivo: Medir el coste medio que supone un servicio eficaz, con respecto a los documentos ofrecidos.

- Campo de aplicación: En principio, todos los SABDL.

- Definición: Gasto directo del SABDL dividido entre el número de registros pertinentes ponderados, quedando definido el gasto directo del SABDL como en el indicador $1 \mathrm{y}$ los registros pertinentes como en el indicador 8 .

- Método: Se obtienen el gasto directo del SABDL en un año natural (véase el indicador 1), el número de registros pertinentes en un año natural (véase el indicador 8), el número total de registros obtenidos en las búsquedas y el número de registros calificados por los usuarios. El valor del indicador es $(\mathrm{AxD}) /(\mathrm{BxC})$, siendo A el gasto directo del SABDL, B el número de registros pertinentes, $\mathrm{C}$ el número total de registros obtenidos en las búsquedasy $\mathrm{D}$ el número de registros calificados por los usuarios.

- Interpretación: En el análisis de la evolución de este indicador, valen las consideraciones realizadas para el indicador 10, en el sentido de que es preferible la vía de aumentar la eficacia sobre la de reducir los costes.

- Fuentes (6): King Research Ltd. 1995 *** (Coste medio del nivel de relevancia de la respuesta), Massísimo $1994 * * *$ (Coste por consulta en cada nivel de satisfacción), Cram $1993 * * * *$ (Cost by satisfaction levels: average input costs compared with levels of satisfaction), Lancaster 1996 ****

\subsection{Gasto por consulta}

- Objetivo: Medir el gasto medio por consulta.

- Campo de aplicación: En principio, todos los SABDL. 
- Definición: Gasto directo del SABDL dividido entre el número de consultas. El gasto directo del SABDL se define como en el indicador 1.

- Método: Se obtienen el gasto directo del SABDL en un año natural (véase el indicador 1) y el número de consultas en un año natural. El valor del indicador es A/B, siendo A el gasto directo del SABDL y B el número de consultas.

- Interpretación: Parece interesante la comparación transversal con el valor de este indicador en otros SABDL, como medio para detectar posibles disfunciones. También puede ser interesante la desagregación por unidades universitarias, por bases de datos consultadas o por tipo de gasto (suscripción, conexión, documentos...).

- Fuentes (6): Abbott 1994 * (Unit cost per search), King Research Ltd. $1995 *$ (Coste de cada búsqueda en línea), Massísimo $1994 *$ (Coste por consulta a base de datos), Cram $1993 * *$ (cost per output).

\subsection{Gasto por registro}

- Objetivo: Conocer el gasto medio por unidad de información ofrecida.

- Campo de aplicación: En principio, todos los SABDL.

- Definición: Gasto directo del SABDL dividido entre el número de registros suministrados. El gasto directo del SABDL se define como en el indicador 1.

- Método: Se obtienen el gasto directo del SABDL en un año natural (véase el indicador 1) y el número de registros suministrados en un año natural. El valor del indicador es $\mathrm{A} / \mathrm{B}$, siendo A el gasto directo del SABDL y B el número de registros suministrados.

- Interpretación: Valen las comparaciones y desagregaciones apuntadas para el indicador 12. Este indicador puede ser valioso como dato objetivo para hacer balance del servicio o para fijar un precio a las consultas.

- Fuentes (6): Massísimo 1994 * (Coste por referencia), King Research Ltd. $1995 *$ (Coste por referencia recuperada), Cram $1993 * *$ (Cost per output)

\subsection{Consultas por bibliotecario}

- Objetivo: Conocer la productividad del servicio respecto al personal que lo atiende.

- Campo de aplicación: En principio, todos los SABDL

- Definición: Número de consultas atendidas por el servicio dividido entre el número de personas que atienden el servicio.

- Método: Se obtienen el número de consultas atendidas por el servicio en un año natural y el número de bibliotecarios que atienden el servicio. El valor del indicador es $\mathrm{A} / \mathrm{B}$, siendo $\mathrm{A}$ el número de consultas y $\mathrm{B}$ el número de bibliotecarios. 
- Método alternativo: Para aquellos SABDL en los cuales el personal que lo atiende no lo hace de manera exclusiva, se deberá ponderar el indicador según el tiempo que absorbe la dedicación al servicio.

Para ello, se obtienen el número de consultas atendidas por el servicio en un año natural, el número de bibliotecarios que atienden el servicio, el tiempo efectivo de las consultas (véase el indicador 6) y el tiempo total de trabajo de los bibliotecarios encargados del servicio (véase el indicador 6). El valor del indicador es $(\mathrm{AxD}) /(\mathrm{BxC})$, siendoA el número de consultas, $\mathrm{B}$ el número de bibliotecarios, $\mathrm{C}$ el tiempo efectivo de las consultas y $\mathrm{D}$ el tiempo total de trabajo de los bibliotecarios encargados del servicio.

- Interpretación: Este indicador muestra la carga de trabajo que soporta (o podría soportar, si se dedicara exclusivamente) cada bibliotecario encargado del servicio. Respecto a su interpretación, la productividad, por sí misma, tiene un valor relativo: una productividad alta, si está acompañada de valores bajos en los indicadores de eficacia, no sería una situación positiva. Por lo tanto, este indicador se debe relacionar con los de eficacia (indicadores 7,8 y 9), para obtener una visión más completa del servicio. Lo ideal sería un equilibrio entre eficacia y productividad.

- Fuentes (6): Abbott $1994 *($ Number of searches conducted per relevant member of staff)

\section{Conclusión}

Del análisis de la bibliografía profesional, se llega a la conclusión de que existe un cierto vacío en el campo de la evaluación de los SABDL. No existe un conjunto consistente de indicadores que permitan evaluar en su totalidad un SABDL, y, en el caso de España, tampoco conocemos un modelo de evaluación integral de un servicio bibliotecario.

La propia norma UNE 50137, en su introducción, indica que "todavía faltan indicadores fiables y bien documentados para algunas actividades y servicios de las bibliotecas. Estos incluyen servicios de información, formación de usuarios y servicios electrónicos en general. [...] Se recomienda a los bibliotecarios y especialistas en información que den prioridad al establecimiento de los mecanismos necesarios para desarrollar indicadores relevantes". Con esta idea, nuestra propuesta pretende realizar una contribución en estas dos líneas: presentar un modelo de evaluación del rendimiento de un servicio y aportar unos indicadores de rendimiento para un SABDL.

Nuestro modelo está pensado para la evaluación sistemática del rendimiento de un servicio concreto de acceso a bases de datos en línea (el de la Biblioteca de la Universidad Pública de Navarra); y por tanto circunscrito a unos determinados 
condicionantes. Sin embargo, se ha intentado adaptarlo, en la medida de lo posible, para que sea válido para cualquier SABDL.

Una de las mayores dificultades que hemos encontrado al componer los indicadores ha sido el determinar pautas de interpretación. Es complicado poder llegar a interpretaciones que sean unívocas en todas las situaciones y en todos los casos. Los diferentes objetivos que pueden tener los SABDL, la inexistencia de paradigmas válidos sobre los indicadores de rendimiento y lo indeterminado de algunos conceptos han motivado que, en algunos casos, no podamos ofrecer mucho más que orientaciones generales, basadas más en el sentido común que en la investigación o la experiencia. Por otra parte, parece aconsejable un seguimiento durante cierto tiempo para determinar la verdadera validez del modelo, e incluso para modificarlo, si así lo aconsejan los resultados obtenidos.

No obstante este origen limitado y estas cauciones en lo que respecta a su validez real, sí nos gustaría que se considerase como una propuesta general, abierta a posteriores contribuciones o modificaciones, para evaluar servicios de acceso a bases de datos en línea.

\section{Notas}

(1) Puede consultarse una reciente revisión bibliográfica en Pinto (1999). Otras recopilaciones de interés pueden verse en Myers (1997), DECIMAL (1995), Ward (1995, p.165-172), Poll (1998, p. 155-209), Abad (1997, p. 193-223), Cotter (1999), Pla d'avaluació ... (1999), Ryan (2000).

(2) Rebiun publica anualmente su Informe estadístico anual de las bibliotecas universitarias y científicas españolas (Rebiun, 2000). Recientemente, en mayo de 2000, el Grupo de Evaluación de Rebiun dio a conocer el documento Indicadores de rendimiento para la evaluación de bibliotecas universitarias (Grupo de Evaluación de Rebiun, 2000).

(3) CAMILE es una acción concertada de la Comisión Europea dedicada a la gestión de información en las bibliotecas de Europa, dirigida a la medición de resultados y al apoyo a la toma de decisiones, y destinada a promover la difusión de los resultados de cuatro proyectos del programa de bibliotecas de la Comisión Europea: EQLIPSE (Evaluation and Quality in Library Performance : System for Europe), MINSTREL (Management Information Software Tool - Research in Libraries), DECIMAL (Decision Making in Libraries) y DECIDE (Decisión Support Models and DSS for European Academic and Public Libraries). Puede consultarse una revisión de estos programas en Clarke (2000).

(4) El modelo que presentamos es una adaptación a un SABDL del modelo general de C. Abbott, que define un conjunto de catorce indicadores esenciales, dentro de un esquema conceptual que posteriormente aplica a una serie de servicios seleccionados. Para el caso del servicio de "mediated on-line searching" propone nueve indicadores.

(5) Los indicadores están planteados para el supuesto de que se recojan todas las consultas. En caso de realizar muestreos, los totales se entenderán como totales del muestreo.

Scire. $7: 1$ (en.-jun. 2001) 89-114. 
(6) Organizadas de la siguiente forma: (*) fuente que documenta el indicador para un servicio similar, $(* *)$ fuente que documenta el indicador para la biblioteca en general, $(* * *)$ fuente que documenta el indicador para servicios bibliotecarios distintos del SABDL, y $(* * * *)$ lectura complementaria que suministra información utilizada para la construcción del indicador. En las referencias de tipo *, ** y *** se recoge, entre paréntesis, el nombre original con el que aparece el indicador.

(7) Es adaptación de un cuestionario de Lancaster (1996, p. 205).

(8) Algunos trabajos han indicado un 55\% de éxito como la media para algunos tipos de transacciones de referencia (se llega a hablar incluso de una "regla del 55\%"). A pesar de ser el único baremo concreto propuesto, ha sido ampliamente contestado. Las especiales características de los productos del SABDL y el no ser un paradigma universalmente admitido, aconsejan no aplicar esta "regla" a la evaluación de los SABDL. Para esta y otras tasas de éxito en servicios de referencia, véanse, por ejemplo, Baker (1991), Mendelsohn (1997), Stalker (1996), Bunge (1987), Allen (1995) y Murfin (1995).

\section{Referencias}

Abad García, María Francisca (1997). Investigación evaluativa en documentación : aplicación a la documentación médica. València : Universitat de València, 1997.

Abbott, Christine (1994). Performance measurement in library and information services. London : ASLIB, 1994.

ACRL (1994). Normas para bibliotecas universitarias: evaluación de la eficacia. // Boletin de la Anabad. 44 : 3 (julio-septiembre 1994) 193-222.

Allen, Bryce (1995). Evaluation of reference services. // Bopp, Richard E. ; Smith, Linda C. Reference and information services : an introduction, 2nd ed. Englewood, CO : Libraries Unlimited, 1995. P. 207-229.

Baker, Sharon L. ; Lancaster, F. Wilfrid (1991). The measurement and evaluation of library services, 2nd ed. Arlington, VA : Information Resources Press, 1991.

Benchmarking Team, University of Virginia Library (1999). Report on benchmarking process : January-May 1999. URL: 〈http://www.lib.virginia.edu/staff/management-information/bench-ProcessRept.html>. Consulta: 16.10.2000.

Blagden, John (1997). Concerted Action on Management Information for Libraries in Europe (CAMILE): key themes for the dissemination programme. April 1997. URL: <http://www.staff.dmu.ac.uk/ camile/JBCamile.html>. Consulta: 16.10.2000.

Boyd, Fraser J. (1997). The customer may be always right-but who is the customer? // Records Management Quarterly. 31 : 2 (April 1997) 38-44.

Brophy, Peter. EQLIPSE: Evaluation and Quality in Library Performance: System for Europe. Preston (England): University of Central Lancashire, Centre for Research in Library and Information Management, 1997. URL: <http://www.mmu.ac.uk/h-ss/cerlim/reports/e_d7_com.htm>.Consulta: 17.10.2000.

Buchanan, H.S. ; Marshall, J.G. (1996). Benchmarking reference services: step-by-step. // Medical Reference Services Quarterly. 15 : 1 (Spring 1996) 1-13. 
Clarke, Zoë ; Lambert, Siân. Management information and decision support for libraries in Europe: a concerted action. // Performance Measurement and Metrics. $1: 2$ (August 2000) 77-98.

Coleman, V ; Xiao, Y. (1997) Toward a TQM paradigm : using SERVQUAL to measure library service quality. // College and Research Libraries. 58 : 3 (May 1997) 237-251.

Cotta-Schønberg, Michael (1995). Performance measurement in the context of quality managemenet. // Proceedings of the 1st Northumbria International Conference on Performance Measurement in Libraries and Information Services. Newcastle Upon Tyne : Information North, 1995. P. 51-62.

Cotter, Rosemary et al. (1999). CAVAL Reference Interest Group (CRIG) : Working Party on Performance Measures for Reference Services : final report, December 1998. Victoria (Australia) : Cooperative Action by Victorian Academic Libraries, 1999. URL: 〈http://www.caval.edu.au/wppmfirp.pdf>. Consulta: 4.10.2000] y URL: <http://home.vicnet.net.au/ caval/wppmfirp.pdf>. Consulta: 4.10.2000.

Cram, Jennifer K. (1993) Measuring quality and productivity : performance measures for non-school libraries. 1993. URL: 〈http://qednsl.qld.gov.au/PERFMEAS.HTML〉. Consulta: 16.10.2000.

Crawford, John; Riley, Sarah (2000). The ESEP Project at Glasgow Caledonian University and the implications for LIS evaluation. // Performance Measurement and Metrics. 1 : 1 (April 2000) 55-70.

Cullen, Rowena (1999). Does performance measurement improve organisational effectiveness? : a postmodern analysis. // Performance Measurement and Metrics. Sample issue (August 1999) 9-30. URL: 〈http://www.aslib.co.uk/pmm/volume/number/01.pdf〉. Consulta: 17.10.2000.

De la Mano González, Marta (1998). Propuesta de un sistema de evaluación para bibliotecas universitarias. // Revista Española de Documentacion Científica. 21 : 2 (abril-junio 1998) 174-197.

DECIMAL : Decision-making in libraries: decision research for the development of integrated library systems, Project LIB-DECIMAL/4-3012. 1995. URL: <http://www.mmu.ac.uk/h-ss/dic/research/decimal.htm>. Consulta: 17.5.2000.

Duarte Barrionuevo, Miguel. Indicadores como instrumento de evaluación de los servicios bibliotecarios : 1, aspectos metodológicos. // Boletín de la Anabad. 45 : 1 (enero-marzo 1995) 95-106.

Fuentes, Juan José. Evaluación de bibliotecas y centros de documentación e información. Gijón : Trea, 1999.

García-Morales Huidobro, Elisa (1995). Aportaciones de la gestión de calidad a bibliotecas y servicios de documentación. // Revista Española de Documentación Científica. 18 : 1 (enero-marzo 1995) 9-18.

Gorman, Gary E. (1999). Collecting Data Sensibly in Information Settings. // 65th IFLA Council and General Conference 1999. URL: <http://ifla.inist.fr/IV/ifla65/papers/004120e.htm>. Consulta: 17.10.2000.

Grupo de Evaluación de Rebiun (2000). Indicadores de Rendimiento para la Evaluación de Bibliotecas Universitarias : mayo 2000. Rebiun, 2000 [inédito. 
Joint Funding Council's Ad-Hoc Group on Performance Indicators for Libraries (1999). La biblioteca universitaria eficaz : directrices para la evaluación del rendimiento en bibliotecas universitarias : informe consultivo para HEFCE, SHEFC, HEFCW y DENI. Cádiz : Universidad, 1999.

Kettinger, William J. ; Lee, Choong C. (1997). Pragmatic perspectives on the measurement of information systems service quality. // MIS Quarterly. 21 : 2 (June 1997) 223-240.

King Research Ltd. (1995). Claves para el éxito : indicadores de rendimiento para bibliotecas públicas. Barcelona : Eumo, 1995.

Lancaster, F.W. (1996). Evaluación de la biblioteca. Madrid : Anabad, 1996.

Lindauer, Bonnie Gratch (2000). Definición y medida del impacto de las bibliotecas universitarias sobre los resultados globales de la institución. // Boletín de la Asociación Andaluza de Bibliotecarios. 59 (junio 2000) 35-75.

Marshall, J.G. ; Buchanan, H.S. (1995). Benchmarking reference services: an introduction. // Medical Reference Services Quarterly. 14 : 3 (Fall 1995) 59-73.

Massísimo, Ángels (1994). Avaluació de serveis de referència. // Ítem. 15 (1994) 104-117.

Mendelsohn, J. (1997). Perspectives on quality of reference service in an academic library : a qualitative study. // RQ. 36 : 4 (Summer 1997) 544-557.

Murfin, M.E. (1995). Evaluation of reference service by user report of success. // Reference Librarian. 49-50 (1995) 229-241.

Myers, Barry L. et al. (1997). A comprehensive model for assessing the quality and productivity of the information system function : toward a contingency theory for information system assessment (ISRC-WP-19970101). University of North Texas Information Systems Research Center, 1997. URL: <http://courses.unt.edu/kappelman/aboutwork/misfiles/articles/page-links/framisrc.htm>. Consulta: 4.10.2000.

Nitecki, Danuta (1997). SERVQUAL : measuring service quality in academic libraries. Association of Research Libraries, 1997. URL: <http://www.arl.org/newsltr/191/servqual.html>. Consulta: 16.10.2000.

Pinto Molina, María ; Suárez, Juana María (1999). Recursos informativos sobre gestión de calidad en bibliotecas y centros de documentación. // Revista General de Información y Documentación. 9 : 1 (1999) 217-273.

Pitt, Leyland F. et al. (1995). Service quality : a measure of information system effectiveness. // MIS Quarterly. 19: 2 (June 1995) 173-187.

Pitt, Leyland F. et al. (1997). Measuring information system service quality : concerns for a complete canvas. // MIS Quarterly. 21 : 2 (June 1997) 209-221.

Pla d'avaluació de la qualitat del Servei de Biblioteca i Documentació [Universitat de les Illes Balears. (1999) Març de 1999. URL: <http://www.uib.es/secc6/biblioteca/secc2/ avaluacio.htm>. Consulta: 19.1.2000.

Poll, Roswitha ; Boekhorst, Peter te (1998). Medición de la calidad : directrices internacionales para la medición del rendimiento en las bibliotecas universitarias. Madrid : Anabad, 1998.

Rebiun (2000). Informe estadístico anual de las bibliotecas universitarias y científicas españolas. 2000. URL: <http://www.uma.es/rebiun/informeestadistico.html>. Consulta: 19.1.2000.

Scire. $7: 1$ (en.-jun. 2001) 89-114. 


\section{Luis Puente, Carlos del Campo y Manuel Ruiz de Luzuriaga}

Ryan, Joe (2000). Library statistics \& performance measures. 2000. URL: <http://web.syr.edu/ jryan/infopro/stats.html>. Consulta: 4.10.2000.

Salgueiro, Amado (1998). Planificación : el arte de establecer objetivos. Madrid : Aenor, 1998.

SCONUL (1992). Performance indicators for university libraries : a practical guide. London : SCONUL, Advisory Committee on Performance Indicators, 1992.

Scriven, Michael (1991). Evaluation thesaurus : fourth edition. London : Sage Publications, 1991.

Spiller, David (1998). Benchmarking in practice. // Proceedings of the 2nd Northumbria International Conference on Performance Measurement in Libraries and Information Services [...] 7 to 11 September 1997. Newcastle upon Tyne: Information North, 1998. P. 431-439.

Stalker, J.C. ; Murfin, M.E. (1996). Quality reference service : a preliminary case study. // Journal of Academic Librarianship. 22 : 6 (November 1996) 423-429.

$\mathrm{Su}$, Louise T. Evaluation measures for interactive information retrieval. // Information Processing and Management. $28: 4$ (July-August 1992) 503-516.

$\mathrm{Su}$, Louise $\mathrm{T}$. Value of search results as a whole as the best single measure of information retrieval performance. // Information Processing and Management. 34 : 5 (September 1998) 557-579.

UNE 50137:2000. Información y documentación : Indicadores de rendimiento bibliotecario. Madrid : Aenor, 2000. [Equivalente a ISO 11620:1998.

UNE-EN ISO 2789:1996 - Información y documentación : Estadísticas internacionales de bibliotecas. Madrid : Aenor, 1996.

Van House, Nancy A. et al. (1990). Measuring academic library performance : a practical approach: prepared for the Association of College and Research Libraries, Ad Hoc Committee on Performance Measures. Chicago, IL ; London : American Library Association, 1990.

Ward, Suzanne et al. (1995). Library performance indicators and library management tools. Luxembourg : European Commission, DG XIII-E3, 1995.

Watson, Richard T. et al. (1998). Measuring information system service quality : lessons from two longitudinal case studies. // MIS Quarterly. 22 : 1 (March 1998) 61-79.

White, Lynda S. (1998). Spring 1998 SERVQUAL Survey. University of Virginia Library, 1998. URL: <http://www.lib.virginia.edu/staff/management-information/sq981.html>. Consulta: 16.10 .2000 .

Wisniewski, Mik; Donnelly, Mike (1996). Measuring service quality in the public sector : the potencial for SERVQUAL. // Total Quality Management. $6: 7$ (August 1996) 357-365.

Zeithaml, Valarie A. et al. (1992). Calidad total en la gestión de servicios : cómo lograr el equilibrio entre las percepciones y las expectativas de los consumidores. Madrid : Díaz de Santos, 1992. 